Orinoquia, Julio-Diciembre 2019;23(2):123-129

ISSN electrónico 2011-2629.

ISSN impreso 0121-3709.

https://doi.org/10.22579/20112629.575

\title{
El compostaje, una alternativa para el aprovechamiento de residuos orgánicos en las centrales de abastecimiento
}

\author{
Composting, an alternative for the use of organic residues In the \\ supply centers
}

\section{Compostagem, uma alternativa para o uso de resíduos orgânicos no suprimento central}

Oscar I Vargas-Pineda ${ }^{1 *}$, Juan M Trujillo-González ${ }^{2 *}$, Marco A Torres-Mora ${ }^{3 *}$

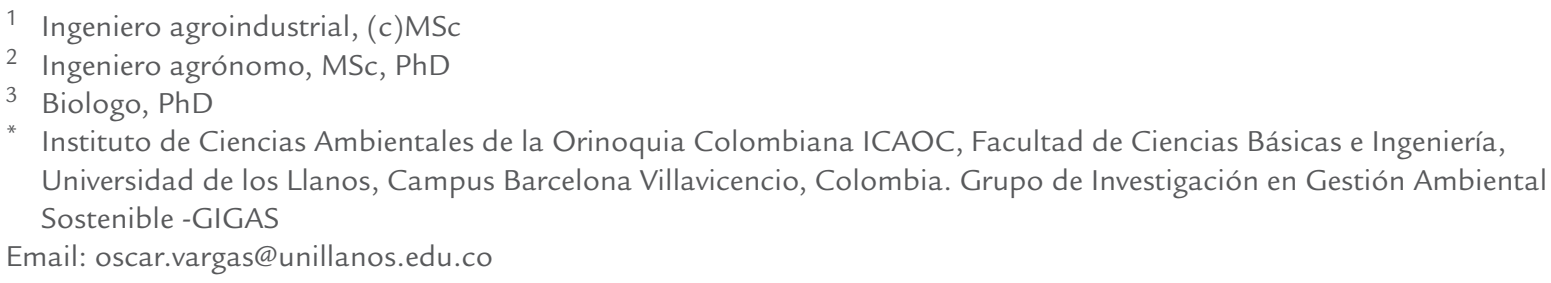

\begin{abstract}
Resumen
La pérdida de alimentos a lo largo de la cadena de suministro se ha convertido en un tema de gran interés en el mundo, debido a que afecta la seguridad alimentaria de la sociedad. Uno de estos eslabones de la cadena de distribución de alimentos son las centrales de abasto local, donde diariamente se pierden importantes cantidades de alimentos especialmente por carencia de protocolos de poscosecha. El objetivo del presente estudio consistió en analizar la pérdida de alimentos en la central de abastos del municipio de Acacias, Colombia y evaluar el sistema de compostaje como alternativa de gestión ambiental. Se realizó mediante el método de cuarteo la estimación de la cantidad y tipo de residuos desperdiciados, que posteriormente se sometieron al proceso de compostaje donde se evaluó la calidad del producto. En conclusión, los residuos orgánicos son un problema ambiental que requiere de alternativas como el compostaje, que permite reducir la cantidad y aprovechar el contenido nutricional de la fracción orgánica, generando subproductos con alto valor agregado.
\end{abstract}

Palabras clave: Residuo orgánico; compostaje; gestión de residuos; producción limpia

\footnotetext{
Abstract

The loss of food along the supply chain has become a topic of great interest in the world, because this affect to the food security of society. One of these stages in the food distribution chain are the local supply centers, where important quantities 
of food are often lost, especially due to the lack of post-harvest protocols. The objective of this study was to analyze the loss of food in the supply center of the municipalities of Acacias, Colombia and evaluate the composting system as an alternative for environmental management. It was carried out by means of the quartering method the estimation of the quantity and type of residues lost, and subsequently, the composting process, where the quality of the product is evaluated. In conclusion, organic residues is an environmental problem that requires alternatives such as composting, which allows to reduce the amount and take advantage of the nutritional content of organic matter, generating by-products with high added value.

Key words: Organic residues; composting; management of residues; clean production

\begin{abstract}
Resumo
A perdida de alimentos ao longo da cadeia de fornecimento converteu-se em um tema de grande interesse no mundo, como isto afeta a segurança alimentar da sociedade. Um destes elos da cadeia de distribuição de alimentos são as centrais de abastecimento locais, onde diariamente se perdem importantes quantidades de alimentos especialmente por carência de protocolos de pós-colheita. O objetivo do presente estudo consistiu em analisar a perdida de alimentos na central de abastecimento do município de Acacias, Colômbia e avaliar o sistema de compostagem como alternativa de gerenciamento ambiental. Realizou-se mediante o método de cuarteo a estimativa da quantidade e tipo de resíduos perdidos, que posteriormente se submeteram ao processo de compostagem onde se avaliou a qualidade do produto. Em conclusão, os resíduos orgânicos são um problema ambiental que requer de alternativas como o compostagem, que permite reduzir a quantidade e aproveitar o conteúdo nutricional da fração orgânica, gerando subprodutos com alto valor agregado.
\end{abstract}

Palavras chave: Resíduos orgânicos; compostagem; gestão de resíduos; produção limpa

\section{Introducción}

La pérdida o desperdicio de alimentos a través de la cadena de suministro, es un tema que cada vez tiene mayor relevancia en el mundo (Cerda et al., 2018), teniendo en cuenta que esta situación altera negativamente la disponibilidad local de alimentos y compromete así la seguridad alimentaria de la sociedad (Basso et al., 2016). Según reportes de la FAO (2011), anualmente se desperdician 1.300 billones de toneladas de alimentos en el mundo, lo que representa el 33\% de la oferta de alimentos disponible para consumo humano, de los cuales el 50\% son productos hortofrutícolas, $30 \%$ cereales y $20 \%$ productos pecuarios (Marmolejo et al., 2010; Caicedo y Ibarra, 2016), alimentos que finalmente se convierten en residuos orgánicos, material que al no ser valorizado, disminuye su valor económico en el mercado comercial (Vargas et al., 2017; Xu et al., 2018). De acuerdo a Sepúlveda (2006), en Colombia el $18 \%$ de estos residuos son generados en las centrales de abastecimiento de mercado y tienen como destino final los rellenos sanitarios, donde se disminuye el potencial de aprovechamiento y valor agregado de otros residuos sólidos, a la vez que aumentan la tasa de lixiviados y acortan el tiempo de uso de éstos (López, 2009; Muñoz y Sánchez, 2013; Herrera, 2015). Por esta razón, se han desarrollado estrategias que están enmarcadas en la economía verde que procuran mejorar la gestión de los residuos orgánicos, fomentando su reducción a lo largo de la cadena de suministros (prácticas agrícolas, poscosecha, comercialización y consumo final) (Vargas et al., 2017), además de esta manera disminuir las perdidas y desperdicio, así como la implementación de acciones de manejo de los residuos orgánicos generados, con la intención de aportar un valor agregado desde un enfoque ambiental y económico (Tapia, Laines y Sosa, 2016; Niño et al., 2017).

El compostaje es una tecnología de bajo costo, que garantiza que los residuos orgánicos vinculen sus componentes en el ciclo de la cadena de producción primaria, además permite mejorar las condiciones físico-químicas del suelo y aumenta la productividad de los cultivos (Jaramillo y Zapata, 2008; Otterpohl et al., 2016; Martínez et al., 2016; Pinzón 2017). En este sentido, teniendo en cuenta la problemática originada por los residuos orgánicos en centrales de abasto en Colombia, se planteó para este estudio los siguientes objetivos: I) Estimar los residuos orgánicos y su composición generados en la central de abastecimiento del municipio de Acacias Colombia, II) Evaluar la calidad del compostaje generado a partir de estos residuos como una alternativa de gestión ambiental, que permita mitigar los posibles efectos originados por la acumulación de residuos orgánicos provenientes de alimentos en el ambiente.

\section{Materiales y métodos}

\section{Área de estudio}

El estudio se realizó en la central de abastecimiento de mercado ubicada en el municipio de Acacias, Meta, Colombia. La central cuenta con 352 establecimientos, de los cuales 185 se dedican a diferentes actividades comerciales como: venta de frutas y verduras, 
tubérculos, carne vacuna, pollo y pescado, comidas preparadas y viveres en general; todos responsables de generar residuos orgánicos de acuerdo a la actividad que realizan.

\section{Caracterización de los residuos}

Mediante el método de cuarteo propuesto por Montoya (2012), se realizó la caracterización de los residuos orgánicos procedentes de alimentos en la central de abastecimiento de mercado. Para esto se tomaron como muestra 20 establecimientos a los que se les dotó con bolsas plásticas para la recolección de los residuos generados en los días de mayor comercialización en la central (martes, sábado y domingo). Posteriormente se realizó un cuarteo donde se clasificó y se procedió al pesaje de cada tipo de residuos.

\section{Proceso y evaluación del compostaje}

El material orgánico que se llevó a compostaje se distribuyó en tres (3) pilas de $24 \mathrm{Kg}$, separados a una distancia de un (1) metro con una cubierta plástica para obtener condiciones optimas de temperatura y prevenir el aumento de la humedad del material por precipitación, de tal modo que se desarrollara el proceso de fermentación aerobio adecuado, además permitiera registrar la temperatura de las pilas en su diferentes etapas durante los tres (3) meses de estudio.

El rendimiento del compost se determinó considerando la relación residuos Vs compost obtenido; para tal fin se utilizó la ecuación 1 , donde, $R$ es rendimiento porcentual del compost que se produce (\%), PF es el peso final del compost generado $(\mathrm{kg}), \mathrm{Pl}$ es el peso inicial del material orgánico que entra en el proceso de compostaje $(\mathrm{kg})$.

$$
R=\frac{P F}{P l} \times 100 \%
$$

\section{Análisis químico del compost}

Para evaluar la calidad del nutricional del compost se realizaron los siguientes análisis de laboratorio: el pH se midió con potenciómetro en relación 1:1 compost : agua; la materia orgánica se determinó por el método de Walkley-Black; los macronutrientes como el fósforo mediante el método Bray II, el potasio y magnesio por el método de espectrofotometría de absorción atómica (IGAC, 2006). Los resultados de análisis del compost obtenido (Tabla 3), se evaluaron teniendo en cuenta los valores propuestos por Munévar (2004) en la guía básica de interpretación de resultados sobre análisis de suelos y la Norma Técnica Colombiana NTC 5167.

\section{Resultados y discusión}

\section{Cuarteo de los residuos orgánicos}

La central de abastecimiento de mercado en estudio, dispone para su actividad comercial de 352 establecimientos, de los cuales 185 generan residuos orgánicos de origen vegetal, animal, de alimentos frescos y procesados. A partir de los datos obtenidos en el cuarteo (Tabla 1), se evidencia que se genera una cantidad importante de residuos orgánicos tanto los días ordinarios, como los típicos de mercado.

Los residuos de un establecimiento de la central de abastecimiento que manipula productos orgánicos para su actividad comercial, generan en promedio $14.3 \mathrm{~kg} /$ día, con un máximo de $16.5 \mathrm{Kg}$ los días de mercado y un mínimo de $11.8 \mathrm{~kg} /$ día los días ordinarios. El cuarteo permitió determinar que los establecimientos de venta de hortalizas y los restaurantes aportaron en su correspondiente orden el $82 \%$ y $18 \%$ de los residuos orgánicos generados en la central de abastecimiento de mercado. Considerando que en este tipo de comercialización, los procesos de poscosecha de los productos agrícolas es inadecuado y el re-

Tabla 1. Cuarteo de residuos orgánicos de la central de abastecimiento de mercado.

\begin{tabular}{|c|l|c|c|c|}
\hline \multirow{2}{*}{ Día } & Tipo de local comercial & $\begin{array}{c}\text { Número de } \\
\text { locales }\end{array}$ & $\begin{array}{c}\text { Residuos generados } \\
(\mathbf{K g})\end{array}$ & $\begin{array}{c}\text { Total residuos } \\
\text { (Kg/día) }\end{array}$ \\
\hline \multirow{3}{*}{ Sábado } & Restaurantes & 4 & 42 & \multirow{2}{*}{235} \\
\cline { 2 - 5 } & Venta de hortalizas & 16 & 193 & \multirow{2}{*}{293.6} \\
\hline \multirow{2}{*}{ Martes } & Restaurantes & 4 & 54.6 & \multirow{2}{*}{329} \\
\cline { 2 - 5 } & Venta de hortalizas & 16 & 239 & \\
\hline \multirow{2}{*}{ Domingo } & Restaurantes & 4 & 58.8 & \\
\cline { 2 - 5 } & Venta de hortalizas & 16 & 270.2 & \\
\hline
\end{tabular}


siduo orgánico es un material de fácil deterioro natural (Ali et al., 2014). Tanto así, que el 60\% de los residuos orgánicos generados en el mundo se disponen en los rellenos sanitario sin tratamiento, contribuyendo a un impacto ambiental, generado por bacterias y hongos que incuban patógenos (Martínez et al., 2016; Porras y González, 2016), motivo éste que debe conducir a plantear alternativas para su aprovechamiento. Sin embargo, los residuos deben ser caracterizados por lo menos una vez al año para dimensionar el problema y determinar su potencial uso (Taboada et al., 2009; Montoya, 2012).

\section{Proceso de compostaje}

El proceso de compostaje se desarrolló durante 90 días, tiempo que según Bohorquez et al., (2016), es el adecuado para obtener una alta transformación y baja humedad del material orgánico, como resultado de un eficiente desarrollo de las cuatro (4) etapas del proceso. Así, en la Figura 1, se observa el comportamiento de la temperatura durante estas etapas. En la primera etapa ocurre una fermentación mesófila, que se caracteriza por un aumento en la temperatura del compostaje de $40{ }^{\circ} \mathrm{C}$, la presencia de microorganismos mesófilos que crecen entre los $15{ }^{\circ} \mathrm{C}$ a $35^{\circ} \mathrm{C}$ y que consumen inicialmente los carbohidratos presentes en la materia orgánica (Sánchez et al., 2017). Las tres muestras analizadas alcanzaron temperaturas de 44.6, 44.6 y $43.3{ }^{\circ} \mathrm{C}$ respectivamente. Además, se observó una disminución significativa del volumen de las pilas y una descomposición de los residuos orgánicos, sin presentar olor desagradable.

La segunda etapa es la fermentación termófila que inicia cuando la actividad metabólica microbiana genera reacciones exotérmicas aumentando la temperatura entre los $40{ }^{\circ} \mathrm{C}$ a $60{ }^{\circ} \mathrm{C}$ (Insam y de Bertoldi, 2007). Los compostajes que se realizaron alcanzaron temperatu-

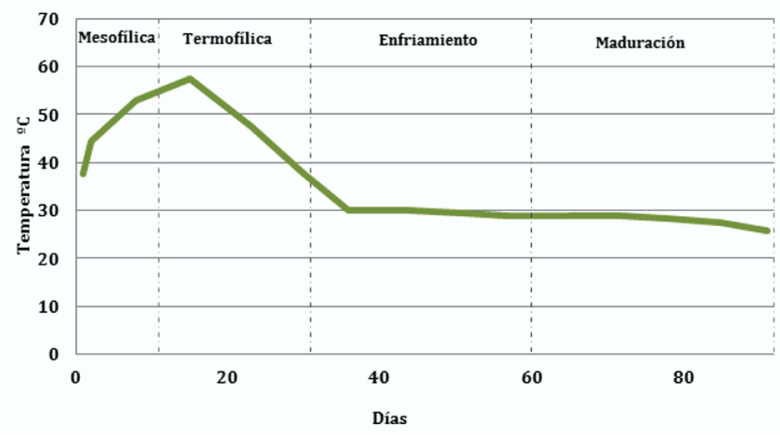

Figura 1. Temperatura promedio del proceso de fermentación aerobio en las fases de compost. ras de $59.2,56.5$ y $54.4{ }^{\circ} \mathrm{C}$ respectivamente, durante la semana dos y tres. Esta condición reduce la actividad de los microorganismos mesófilos mientras aumenta la de los microorganismos termófilos que contienen enzimas que degradan compuestos complejos como las proteínas, además en esta etapa se eliminan organismos patógenos (Neklyudov et al., 2008).

La tercera etapa es el enfriamiento, que inicia con la disminución de temperatura hasta alcanzar la temperatura ambiente, esto ocurrió desde el día 22 hasta el día 90, donde se registraron temperaturas de 28.3, 28.3 y $28.1{ }^{\circ} \mathrm{C}$, como se observa en la Figura 1. Esta disminución se genera por la reducción de energía en el compost y reactivación de los microorganismos mesófilos que descomponen los azucares restantes (Zeng et al., 2010). Por último, en la etapa de maduración, donde los compostajes alcanzaron temperaturas de $25.9,25.8$ y $26.3^{\circ} \mathrm{C}$, se reduce la actividad metabólica. Sin embargo, los compuestos menos degradables se descomponen y surgen los precursores de sustancias húmicas (Vélez-Sánchez-Verín et al., 2008), además de la disminución en un 40\% aproximadamente, del volumen de las pilas.

Asimismo, la materia orgánica presentó una descomposición total, de tal manera que el tamaño de partícula se conformó homogéneo, presentó textura y color similar al suelo, características que indicaron que el proceso del compostaje se desarrolló en condiciones de temperatura, pH y humedad adecuadas, debido principalmente a la correcta aireación que se realiza mediante el volteo de las pilas (Acosta et al., 2012; Montoya et al., 2016). La producción de compost por establecimiento en la central de abastecimiento de mercado fue de $0.75 \mathrm{~kg} /$ día, el rendimiento promedio de la producción de compost fue del $61.7 \%$ con respecto al peso inicial, lo cual concuerda con huerta et al., (2008) y Navia et al., (2013) que expresan un rango de rendimiento entre 50 - 60\%; se considera que el residuo orgánico restante se emite a la atmósfera en forma de vapor de agua y $\mathrm{CO}_{2}$ (Muñoz et al., 2015). El rendimiento de la prueba de manera detallada se presenta en la Tabla 2.

\section{Análisis químico del compost}

El compost óptimo para su aprovechamiento agrícola requiere de parámetros como el pH, materia orgánica, fósforo, magnesio y potasio entre otros, que dimensionan la calidad del producto obtenido del proceso de compostaje (Soliva y Lopez, 2004). Los resultados de análisis del compost obtenido se evidencia en la Tabla 3. 
Tabla 2. Rendimiento en peso de la producción de compostaje obtenido.

\begin{tabular}{|c|c|c|c|}
\hline Ensayo & $\begin{array}{c}\text { Peso residuos } \\
\text { frescos } \mathbf{( k g})\end{array}$ & $\begin{array}{c}\text { Peso compost } \\
(\mathbf{k g})\end{array}$ & $\begin{array}{c}\text { Rendimiento } \\
(\mathbf{\%})\end{array}$ \\
\hline 1 & 24 & 14.8 & 61.6 \\
\hline 2 & 24 & 14.2 & 59.1 \\
\hline 3 & 24 & 15.5 & 64.5 \\
\hline Promedio & 24 & 14.8 & 61.7 \\
\hline
\end{tabular}

El pH que se obtuvo en el compost en promedio fue de 5.6 clasificado como "alto", esto indica que el $\mathrm{pH}$ contribuye a la dinámica de los nutrientes en el compost, teniendo en cuenta que este parámetro se vincula con la disponibilidad de nutrientes (González et al., 2016). $\mathrm{pH}$ ácidos ( $\mathrm{pH}<5.5)$ indican que las condiciones aerobias no fueron óptimas por deficiencias en la aireación o la humedad y se disminuye el contenido de $\mathrm{P}, \mathrm{N}$, y cationes $(\mathrm{Ca}, \mathrm{K})$. Por otro lado, $\mathrm{pH}$ básicos $(\mathrm{pH}>7.3)$ indican el contenido en nitrógeno amoniacal y carbonatos solubles que disminuye la cantidad de micronutrientes (B, Al, Mg, Fe, Co y

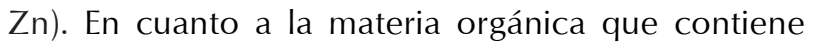
el compost obtenido fue del $27 \%$, clasificado como "medio". Este parámetro depende de la capacidad de descomponer los materiales orgánicos y su porcentaje en el compost, permite mejorar las propiedades fisicoquímicas y biológicas que contribuye a la estabilidad estructural del suelo (Julca et al., 2006). El fosforo en promedio que se obtuvo fue de $119 \mathrm{ppm}$, es un alto contenido de este nutriente que se requiere para el adecuado desarrollo microbiano (Campitelli et al., 2010). El potasio en promedio alcanzó un 3.9 meq/ 100 g clasificado como "medio", no obstante su concentración es aceptable, considerando que es de los nutrientes minerales de mayor requerimiento por parte de la planta en altas concentraciones para su óptimo crecimiento, porque activa enzimas fundamentales en los procesos de fotosíntesis (Becerra et al., 2007; Ansorena et al., 2014). Por último, el magnesio que se registró en el compost fue de 1.26 meq/100 g clasificado como "alto", este nutriente no es asimilado por la planta de manera inmediata; sin embargo, contribuye al cambio en las condiciones del suelo posteriormente (Rodríguez et al., 2010). Finalmente se encontró que las características químicas del compostaje tienen condiciones de calidad adecuadas para mejorar los sistemas de producción agrícola.

\section{Conclusiones}

Los residuos orgánicos que se generan en la central de abastecimiento de mercado del municipio de Acacias - Meta, tienen un potencial de uso agrícola con la transformación de este material a través del compostaje, para lo cual se debe realizar una cuantificación y cualificación en su origen, de tal manera que permita determinar la cantidad y calidad del material orgánico generado para su aprovechamiento. El compostaje se desarrolló adecuadamente teniendo en cuenta la temperatura que se registró en las cuatro etapas del proceso, lo que demostró que las variables de humedad y aireación en el proceso fueron apropiadas, de tal manera que garantizaron un comportamiento aceptable en las cuatro etapas del proceso. Finalmente, la caracterización fisicoquímica del compost obtenido presentó una calidad y rendimiento aceptable, que permite la aplicación de este producto como un abono orgánico en actividades agrícolas, infiriendo así, el compostaje como una herramienta para la gestión ambiental de este tipo de residuos orgánicos.

Tabla 3. Resultado de análisis de caracterización del compost obtenido.

\begin{tabular}{|c|c|c|c|c|c|}
\hline \multirow{2}{*}{ Muestra } & \multicolumn{5}{|c|}{ Parámetros } \\
\cline { 2 - 6 } & $\mathbf{p H}$ & $\begin{array}{c}\mathbf{M . O} \\
(\mathbf{\%})\end{array}$ & $\begin{array}{c}\mathbf{P} \\
(\mathbf{p p m})\end{array}$ & $\begin{array}{c}\mathbf{M g} \\
(\mathbf{m e q} / \mathbf{1 0 0 g})\end{array}$ & $\begin{array}{c}\mathbf{K} \\
(\mathbf{m e q} / \mathbf{1 0 0 g})\end{array}$ \\
\hline 1 & 5.8 & 32 & 144 & 2.30 & 5 \\
\hline 2 & 5.4 & 22 & 100 & 0.70 & 2.6 \\
\hline 3 & 5.5 & 28 & 114 & 0.80 & 4 \\
\hline Promedio & 5.6 & 27 & 119 & 1.26 & 3.9 \\
\hline Calificación & Alto & Medio & Alto & Alto & Medio \\
\hline
\end{tabular}




\section{Referencias}

Acosta Y, Zárraga A, Rodríguez L, El Zauahre M. Cambios en las propiedades fisicoquímicas en el proceso de compostaje de lodos residuales. Multiciencias. 2012;12:18-24.

Ali A, Zahid N, Manickam S, Siddiqui Y, Alderson P. Double layer coatings: New technique for mintainingg physicochemicla chracteristics and antioxidants properties of daragon fruit during storage. Food Bioprocess Technol. 2014;7(8):2366-2374.

Ansorena J, Batalla E, Merino D. 2014. Propiedades fisicoquímicas del compost de residuos de alimentos y su empleo como componente de sustratos. ACTAS DE HORTICULTURA nº $67 \mathrm{XI}$ JORNADAS DE SUSTRATOS. Pp.18.

Basso N, Brkic M, Moreno C, Pouiller P, Romero A. Valoremos los alimentos, evitemos pérdidas y desperdicios. Diaeta. $2016 ; 34(155): 25-32$.

Becerra-Sanabria LA, Navia-de Mosquera SL, Ñústez-López CE. Efecto de niveles de fósforo y potasio sobre el rendimiento del cultivar 'Criolla Guaneña'en el departamento de Nariño. Rev Latinoam Papa. 2007;14(1):51-60.

Bohórquez A, Puentes YJ, Menjivar JC. Evaluación de la calidad del compost producido a partir de subproductos agroindustriales de caña de azúcar. Corpoica. Cienc Tecnol Agropecuaria. 2014;15(1):73-81.

Campitelli P, Aoki A, Gudelj O, Rubenacker A, Sereno R. Selección de indicadores de calidad de suelo para determinar los efectos del uso y prácticas agrícolas en un área piloto de la región central de Córdoba. Ciencia del suelo. 2010;28(2):223-231.

Caicedo NBM, Ibarra AAR. 2017. Estado actual de los niveles de desperdicio de las cadenas de abastecimiento de alimentos. In Memorias de Congresos UTP(pp. 202-209).

Cerda A, Artola A, Font X, Barrena R, Gea T, Sánchez A. Composting of food wastes: Status and challenges. Bioresour Technol. 2018;248(Parte A):57-67.

González A, Szostak J, Morel J, Ishiwata T. Efecto de rotaciones de cultivos en la evolución del contenido de cationes y el pH de suelos arcillosos bajo siembra directa, en el Distrito de Capitán Miranda-Paraguay. Tecnología Agraria. 2016;1(1):51-56.

Herrera LA. 2015. ¿La realidad actual de la ciudad de Villavicencio permitiría la adecuada implementación del decreto 2981 de 2013 en su acápite de separación en la fuente de residuos sólidos ordinarios? (Bachelor's thesis, Universidad Militar Nueva Granada).

Huerta O, López M, Soliva M, Zaloña M. 2008. Compostaje de Residuos Municipales-Control del proceso, rendimiento y calidad del producto. Agència de Residus de Catalunya. CD.

ICONTEC - Instituto Colombiano de Normas Técnicas y Certificación. 2004. Norma Técnica Colombiana. Productos para la industria agrícola. Productos orgánicos usados como abonos o fertilizantes y enmiendas de suelo (pág. 43). Bogotá, Colombia: Instituto Colombiano de Normas Técnicas y Certificación (ICONTEC).
Insam H, Bertoldi M. 2007. Microbiology of the composting process. In: Diaz, L. F., de Bertoldi, M., Bidlingmaier, W., Golueke, C. (Eds.), Compost Science and Technology. Elsevier. pp. 25-48.

IGAC - Instituto Geográfico Agustín Codazzi. 2006. Métodos Analíticos del Laboratorio de Suelos, 6th ed.; IGAC: Bogotá, Colombia, p. 648.

Jaramillo G, Zapata L. 2008. Aprovechamiento de los residuos sólidos orgánicos en Colombia. Monografía para optar el título de Especialistas en Gestión Ambiental. Medellín.Colombia.

Julca-Otiniano A, Meneses-Florián L, Blas-Sevillano R, Bello-Amez S. La materia orgánica, importancia y experiencia de su uso en la agricultura. Idesia (Arica). 2006;24(1):49-61.

López NC. 2009. Propuesta de un programa para el manejo de los residuos sólidos en la plaza de mercado de Cereté-Córdoba (Master's thesis).

Martínez K, Sánchez J, Raga Y, Mármol Z, Arenas E, Mazzarri CA. Cuantificación y caracterización de los residuos de alimentos del comedor central estudiantil de la Universidad del Zulia. Revista Tecnocientífica URU. 2016;8:57-67.

Marmolejo LF, Madera CA, Torres P. Gestión de los residuos sólidos en hospitales locales del norte del Valle del Cauca, Colombia. Rev Fac Nac Salud Pública. 2010;28(1):56-63.

Montoya A. Caracterización de Residuos Sólidos. Cuaderno Activa. 2012;4:67-72.

Montoya A, Soto O, Brieva J. Cinética de aireación para la elaboración de un compost/Aeration kinetics for the preparation of compost. Revista Estudiantil URU. 2016;(2):75-85

Munévar F. Criterios agroecológicos útiles en la selección de tierras para nuevas siembras de palma de aceite en Colombia. Revista Palmas. 2004;25(especial):148-159.

Muñoz AFZ, Sánchez CEZ. Un método de gestión ambiental para evaluar rellenos sanitarios. Gestión y Ambiente. 2013;16(2):105-120.

Muñoz JM, Muñoz JA, Rojas CM. Evaluación de abonos orgánicos utilizando como indicadores plantas de lechuga y repollo en Popayan, Cauca. Biotecnología en el Sector Agropecuario y Agroindustrial (BSAA). 2015;13(1):73-82.

Navia CA, Zemanate Y, Morales S, Prado FA, Albán OÉ. Evaluación de diferentes formulaciones de compostaje a partir de residuos de cosecha de tomate (solanumlycopersicum). Biotecnología en el Sector Agropecuario y Agroindustrial (BSAA). 2013;11(spe):165-173.

Neklyudov AD, Fedotov GN, Ivankin AN. Intensification of composting processes by aerobic microorganisms: a review. Appl Biochem Microbiol. 2008;44:6-18.

Niño-Torres Á, Trujillo-González J, Niño-Torres A. Gestión de residuos sólidos domiciliarios en la ciudad de Villavicencio. Una mirada desde los grupos de interés: empresa, estado y comunidad. Revista Luna Azul. 2017;44:177-187.

Otterpohl R, Grottker M, Lange J. 2016. Gestión sostenible del agua y de los residuos en zonas urbanas. Boletín CF+S. (2). 
Pinzón-Casas RD. Producción, recolección y disposición de residuos sólidos urbanos, análisis del sistema de gestión en el municipio de Puerto Asís, Putumayo.

Porras ÁC, González AR. Aprovechamiento de residuos orgánicos agrícolas y forestales en Iberoamérica. Academia y Virtualidad. 2016;9(2):90-10. DOI: https://doi.org/10.18359/ ravi.2004

Rodríguez-Torres MD, Venegas-González J, Angoa P, MontañezSoto JL. Extracción secuencial y caracterización fisicoquímica de ácidos húmicos en diferentes compost y el efecto sobre trigo. Rev Mexicana Cienc. Agric. 2010;1(2):133-147.

Sepúlveda L. 2006. Aprovechamiento de residuos reciclables en Colombia y en el valle de Aburrá, Cali. OCCIDENTE

Sánchez ÓJ, Ospina DA, Montoya S. Compost supplementation with nutrients and microorganisms in composting process. Waste Manage. 2017; 69:136-153.

Soliva M, López M. 2004. Calidad del compost: Influencia del tipo de materiales tratados y de las condiciones del proceso. En Formación de técnicos para el tratamiento y gestión de lodos de depuradora. Pp.1-20.

Taboada PA, Armijo C, Aguilar Q, Ojeda S, Aguilar, X. 2009. Métodos para la determinación de generación de residuos en comunidades rurales. In Proceedings of the II Simposio Iberoamericano de Ingeniería de Residuos, REDISA, Universidad del Norte, Barranquilla, Colombia. Pp. 554-560.
Tapia-Gómez A, Laines-Canepa J, Sosa-Olivier J. Digestión anaerobia de la fracción orgánica de los residuos sólidos urbanos generados en las cafeterías de la DACBiol. Kuxulkab'. 2016;22(42):512.

Vargas-Pineda Óı, Trujillo-González JM, Torres-Mora MA. Análisis de la inclusión de aspectos ambientales en microempresas agroindustriales de la ciudad de Villavicencio, Colombia. Producción+ Limpia. 2017;12(1):115-123.

Vargas-Pineda Óı, Trujillo-González JM, Torres-Mora MA. La economía verde: un cambio ambiental y social necesario en el mundo actual. Revista de Investigación Agraria y Ambiental (RIAA). 2017;8(2):175-186.

Vélez-Sánchez C, Pinedo-Álvarez C, Viramontes-Oliva O, Ortega C, Melgoza-Castillo A. Bio-tecnologías ambientales para el tratamiento de residuos ganaderos (Environmental biotechnologies for treatment of livestock waste, in Spanish). Creativ Desarr Tecnol. 2008;2:131-144.

Xu F, Li Y, Ge X, Yang L, Li Y. Anaerobic digestion of food waste-Challenges and opportunities. Bioresour Technol. 2018;247:10471058.

Zeng GY, Chen M, Huang Y, Zhang D, Huang J, Jiang H, Yu R. Effects of inoculation with Phanerochaete chrysosporium at various time points on enzyme activities during agricultural waste composting. Bioresour Technol. 2010;101:222-227.

Oscar Vargas: https://orcid.org/0000-0002-6462-4264 Juan Trujillo: https://orcid.org/0000-0001-9612-4080 Marco Torres: https://orcid.org/0000-0002-3824-5412 